\title{
Changes in reference phrases as a function of frequency of usage in social interaction: a preliminary study'
}

\author{
Robert M. Krauss and Sidney Weinheimer \\ BELL TELEPHONE LABORATORIES, INCORPORATED
}

\begin{abstract}
Pairs of subjects interacted in a problem-solving task which required them to communicate about ambiguous figures. The length of the reference phrase for each figure was calculated. A negative relationship was found between the frequency with which a figure was referred to and the mean length of its reference phrase.

\section{Problem}

This paper reports a preliminary study in a program of research on the dynamics of linguistic changes which occur in the course of social interaction. The present study is concerned with the manner in which reference phrases (verbal labels) given to ambiguous figures change during interaction.

We hypothesized that subjects faced with the task of communicating about figures which are unique (in the sense that they do not evoke a common or "popular" reference phrase) would converge upon names for the figures. Further, it was hypothesized that these reference phrases would undergo modifications as a function of the frequency with which the subjects found it necessary to use them.
\end{abstract}

\section{Method}

The subjects' task was to match pairs of cards. Each
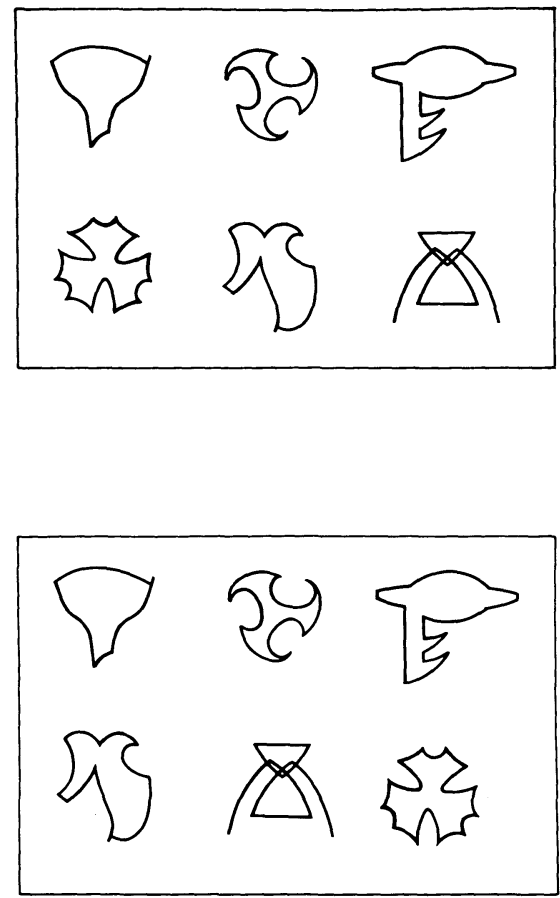

Fig. 1. Two cards from a six-card set. subject had an identical set of six cards, each of which contained six ambiguous figures. In a given set, the same six figures appeared on every card. Three of the six figures (the "redundant figures") appeared in the same position on all cards. The remaining three (the "discriminating figures") appeared in permuted positions. An example of two cards from a set appears in Fig. 1.

One subject's cards were labelled from A through $\mathrm{F}$ and the other's from 1 through 6 . However, the letters and numbers were assigned randomly, so that subjects could not match cards simply on the basis of the identifying labels. Subjects were instructed to determine the correct letter-number matches for identical pairs of cards. Each pair of subjects worked on a total of 16 six-card sets and went through the sets in a different random order.

The six figures which appeared in a given set were drawn from a pool of 15 figures. Each figure had an assigned probability of appearing in a given set as a redundant or discriminating figure. These probabilities ranged from 1.0 to 0.125 .

Three pairs of female and two of male college undergraduates were run. They worked in visually separated soundproof booths and communicated over a highquality audio link. Their conversations were tape-recorded and later transcribed verbatim. The type script was coded by calculating the length (number of words) of each reference to each figure. Coder agreement was high, but because of the small sample size no systematic study of coding reliability was undertaken.

\section{Results}

Figure 2 is a plot of the mean length of reference phrase for each figure as a function of the number of times that figure was referred to. Data for all subjects are shown. Clearly there is a tendency for infrequently mentioned figures to be given reference phrases with long mean lengths and for frequently mentioned figures to be given relatively short reference phrases.

Some data suggesting the process which brings this relationship about appears in Fig. 3. Here we have plotted the mean length of reference phrase for all figures on the first occasion they are mentioned, the second occasion, and so on, up to 25 repetitions. Curves for the five pairs are plotted individually. The first time a figure is referred to the reference phrase tends to be fairly lengthy. But over time with repetition the length rapidly decreases, approaching the lower limit of one word. As an example, one pair of subjects re- 
ferred to a figure on the first mention as an "upsidedown martini glass in a wire stand," then as an "inverted martini glass," then as a " martini glass," and then, after some time, simply as "martini."

\section{Discussion}

Our results quite clearly indicate that the length of a reference phrase used to identify an ambiguous object diminishes as a function of its frequency of usage in the course of social interaction. Given the nature of our subjects' task and the strategy they employed, this result is not surprising. Essentially, the task required that they communicate about the location of the discriminating figures. To do this it was necessary that they develop a means of referring to these figures. The most commonly employed strategy was to assimilate the figures to variations of common objects (e. g., "an upside-down martini glass in a wire stand," "a boomerang with a notch in the blade"). Having identified the figure, it was then possible to "code" the figure's name into a shortened version of the initial reference phrase (e. g., "martini,", "boomerang").

Similar processes may be observed in technical or other sorts of specialized vocabularies. Such shortenings as "hypo" from "hypothiosulfate of soda solution" (among photographers), "comps" from "comprehensive examinations" (among graduate students), and "dikes", from "diagonal cutting pliers" (among elec-

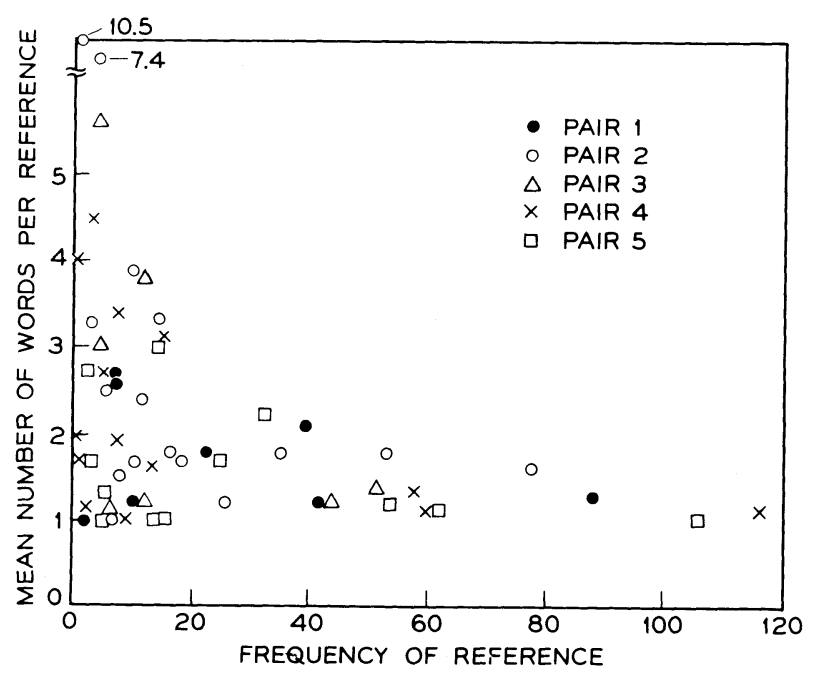

Fig. 2. Mean length of reference phrase as a function of frequency of reference.

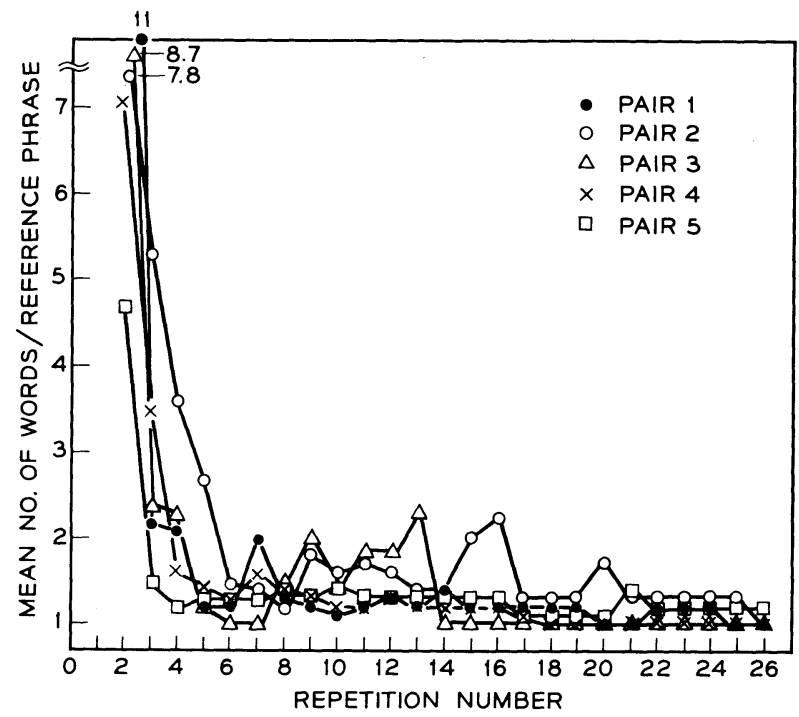

Fig. 3. Mean length of reference phrase on successive repetitions.

tronics technicians) are common examples.

Some similarity may be noted between Zipf's (1935) finding, that for natural languages a negative relationship exists between the size of a word and its frequency of usage, and the results of the present study. One would expect that the processes of linguistic change in a large linguistic community are both more subtle and more complex than those observed in our experimental situation. However, studies such as the present one may shed some light upon the dynamics which produce Zipf's findings.

Because of the small number of subjects employed and the preliminary nature of this study, these findings should be regarded as tentative. A full-scale study attempting to replicate these findings with a larger sample and some additional experimental variations is presently under way. We are concurrently running a study investigating the effect of types of feedback on changes of reference phrases.

\section{Reference}

ZIPF, G. K. The psycho-biology of language. Boston: Houghton Mifflin, 1935.

\section{Note}

1. We gratefully acknowledge the aid of Mrs. Freda Zeiter, who devised the figures used in this experiment, and the technical advice and comments of P. D. Bricker. 